\title{
Modelling and analysis of population dynamics using Lur'e systems accounting for competition from adult conspecifics
}

\author{
Eric Alan Eager ${ }^{a, b}$ \\ ${ }^{a}$ Mathematics Department, University of Wisconsin-La Crosse, La Crosse, WI, USA; ${ }^{\mathrm{b}}$ River Studies Center, La \\ Crosse, WI, USA
}

\begin{abstract}
We study the equilibrium dynamics of a Lur'e system modelling a structured population, where adult conspecifics are assumed to have a negative density-dependent feedback on the recruitment of possible recruits. We find that, depending on the model's parameter values, the population either goes extinct or has a positive equilibrium that is asymptotically stable, globally attracting or globally asymptotically stable. We apply our results to an integral projection model for the Platte thistle (Cirsium canescens) and highlight open aspects of this problem for future work.
\end{abstract}

\section{ARTICLE HISTORY}

Received 7 October 2015

Accepted 25 February 2016

\section{KEYWORDS}

Lur'e systems; population dynamics; structured population models; competition; density-dependence; stability

\section{Introduction}

Population dynamics is the area of science which attempts to explain the time variations of the size and structure of biological populations in a simple, mechanistic way (Bacaer, 2011). When modellers drop the assumption of homogeneity within the population, a structured population model is used. Structured population models describe the evolution and distribution of populations of individuals throughout different classes, categories or characteristics through modelling the contributions of, and/or interactions between, individuals within and throughout these stages. For example, the categorization of individuals can be based upon age, measure of body size, life cycle stage, spatial location and gender or genetic differences (Caswell, 2001; Cushing, 1998; Easterling, Ellner, \& Dixon, 2000). Structured models have the advantage of being able to create a link between the individual level and the population level, accounting for dynamical behaviours that simple, unstructured models cannot.

It is common for population biologists to use discrete-time models to model stagestructured populations, which generally take the form of difference equations. Population projection matrix (PPM) models, which assume that the population is structured with respect to a discrete stage, are commonly used for predicting the dynamics of structured populations in discrete time (for a survey of PPMs, see Caswell, 2001). In many cases, however, the stage used to structure the population is continuous (size, for example).

CONTACT Eric Alan Eager eeager@uwlax.edu 
Instead of discretizing the stage variable so that one can use a PPM population modellers, beginning with Easterling et al. (2000), have started using integral projection models (IPMs), which assume that continuous stages (but still consider time as discrete). Despite the different modelling assumptions, the mathematical properties of PPMs and IPMs are very similar (Lubben, 2009). In this paper, we study discrete time models of the form

$$
n_{t+1}=M\left(n_{t}\right)
$$

where the sequence $\left\{n_{t}\right\}_{t=0}^{\infty}$ evolves in a Banach space $X$ (which is often called the population's state space) and $M$ is a non-negative operator from $X$ to itself. The Banach spaces $X$ we will explicitly consider will be $L^{1}(L, U)$, for some continuous set of stages $(L, U)$ (in the IPM case), or $\mathbb{R}^{m}$ (in the PPM case). Provided the operator $M$ is linear (the ecological processes involved are density independent), the long-term populations grow (or decline) exponentially at a rate of $\lambda$, the leading eigenvalue of $M$ (Lubben, 2009). Many processes in population biology are density dependent, however, which cause the operator $M$ to be nonlinear. When $M$ is nonlinear the population usually does not exhibit exponential growth as $t \rightarrow \infty$. In deterministic, density-dependent models one usually sees the population converge an equilibrium, a cycle, invariant loop or a strange attractor (Caswell, Takada, \& Hunter, 2004).

Many modellers break the operator $M$ into two operators

$$
M=A+B
$$

where the operator $A$ models survival and movement between stages and $B$ models reproduction (Cushing, 1998; Rebarber, Tenhumberg, \& Townley, 2012; Townley, Tenhumberg, \& Rebarber, 2012). The authors Rebarber et al. (2012) and Townley et al. (2012) assume that $A$ is a linear operator, with $B$ a nonlinear (density-dependent) operator and decompose $B$ by assuming that a juvenile's stage variable is independent of its mother's stage variable, and is also independent of population size. This allows $B$ to be written as

$$
B=b F,
$$

where $F$ is a nonlinear functional from $X$ to $\mathbb{R}^{+}$that gives the density of new recruits created by the population each time-step, and $b$ is the juvenile stage distribution. The authors in Rebarber et al. (2012) and Townley et al. (2012) also assume that $F$ can be written as

$$
F\left(n_{t}\right)=g\left(c^{T} n_{t}\right) c^{T} n_{t}=f\left(c^{T} n_{t}\right),
$$

where $f$ is a scalar function from $\mathbb{R}^{+}$to itself and $c^{T}$ is a linear functional from $X$ to $\mathbb{R}^{+}$. Here, $c^{T} n_{t}$ is the density of possible recruits at time $t$ (for example, seeds or seedlings), while $g\left(c^{T} n_{t}\right)$ is the establishment probability of a possible recruit, given a density of $c^{T} n_{t}$ of possible recruits. The establishment probability $g$ is generally a decreasing function of its argument, as an increase in, for example, seed density is assumed to decrease the establishment probability of a any given seed. With these, one can write (1) as

$$
n_{t+1}=A n_{t}+b f\left(c^{T} n_{t}\right)
$$


which is referred to as a Luré system. Luré systems have been used frequently to study the dynamics of plant and fish populations (see, for example, Eager et al., 2012, 2014a, 2014b; Eager \& Rebarber, 2016; Rebarber et al., 2012; Rose et al., 2005; Townley et al., 2012).

As long as the triple $\left(A, b, c^{T}\right)$ satisfies realistic ecological assumptions and $f(y)=g(y) y$ is increasing, concave down, with $f(0)=0$, the long-term dynamics of (2) are determined by the stability radius of $\left(A, b, c^{T}\right)$, which we will call $p_{e} \cdot p_{e}$ is the smallest positive number $p$ such that the linear operator

$$
A+p b c^{T}
$$

has spectral radius equal to 1 . Notice that $A+p b c^{T}$ is just the operator in the model (2) with $f(y)=g(y) y$ replaced with the linear function $f(y)=p y$. Thus, $p_{e}$ is simply the establishment probability that would cause stasis in a density-independent setting.

It is proved in Hinrichsen and Pritchard (2005) that

$$
p_{e}=\left(c^{T}(I-A)^{-1} b\right)^{-1}
$$

If we define $g_{0}:=\sup _{y>0} g(y)$ and $g_{\infty}:=\inf _{y>0} g(y)$ to be the highest and lowest possible density-dependent establishment probabilities, respectively, then it is proven in Rebarber et al. (2012) and Townley et al. (2012) that if the stability radius $p_{e}<g_{\infty}$, the the spectral radius of the operator

$$
A+g(y) b c^{T}
$$

will be greater than unity for all $y$, meaning the population will eventually blow up. On the other hand, if $p_{e}>g_{0}$, the spectral radius of (3) is smaller than unity for all $y$, causing the population to eventually go extinct. If $p_{e} \in\left(g_{\infty}, g_{0}\right)$, the establishment probability is in a region where the population eventually settles down to a globally stable equilibrium vector. The results can be summarized in the following theorem from Rebarber et al. (2012) and Townley et al. (2012):

\section{Theorem 1.1:}

(1) If $p_{e}>g_{0}$, then the zero vector is a globally stable equilibrium for (2) in the sense that for every $n_{0}$ in the positive cone $K$ of $X$,

$$
\lim _{t \rightarrow \infty} n_{t}=0
$$

Furthermore, for every $\epsilon>0$, there exists $\delta>0$ such that $\left\|n_{0}\right\|<\delta$ implies $\left\|n_{t}\right\|<\epsilon$ for all $t \in \mathbb{N}$.

(2) If $p_{e} \in\left(g_{\infty}, g_{0}\right)$ then there exists $y^{*}$ which satisfies the equation $f\left(y^{*}\right)=p_{e} y^{*}$. The vector $n^{*} \in X$ given by

$$
n^{*}=p_{e} y^{*}(I-A)^{-1} b
$$

is a globally asymptotically stable equilibrium of (2) on $K \backslash\{0\}$, i.e.

$$
\lim _{t \rightarrow \infty} n_{t}=n^{*}
$$

and for every $\epsilon>0$, there exists $\delta>0$ such that $\left\|n_{0}-n^{*}\right\|<\delta$ implies $\left\|n_{t}-n^{*}\right\|<\epsilon$ for all $t \in \mathbb{N}$.

(3) If $p_{e}<g_{\infty}$, then there exists $n_{0} \in K$ such that $\lim _{t \rightarrow \infty}\left\|n_{t}\right\|=\infty$. 
One may notice that $p_{e}$ is the inverse of the inherent net reproductive number (see Cushing, 1998, p. 7) for the linear operator

$$
A+1 b c^{T}
$$

the operator from (2) when one assumes that all possible recruits establish (i.e. $g(y)=1$ for all $y$ ). The inherent net reproductive number, which we will denote with $n_{R}$, is the expected number of offspring per newborn during the duration of its lifetime, and for the operator (4) it is the expected number of possible recruits per newborn during the duration of its lifetime. Notice, in this formulation, it follows that $p_{e} n_{R}=1$ is the average number of newborns produced during the lifetime of the average newborn once the population is at equilibrium. From this perspective, the population modelled by (2) blows up if $n_{R} g_{\infty}>1$, goes extinct if $n_{R} g_{0}<1$ and has a globally stable equilibrium if $n_{R} g_{0}<1<n_{R} g_{\infty}$. Biologically, the first case depicts the situation where the average newborn has at least one offspring in its lifetime, even when the establishment probability is at its lowest, while the second depicts the case where the average newborn has fewer than one offspring, even when establishment probability is at its highest, and the third case posits the existence of an establishment probability such that the average number of offspring per newborn will be equal to one. While the results in this paper can be reformatted in the context of $n_{R}$, we prefer to work with $p_{e}$, since it provides a clear threshold for which the densitydependent establishment probability $g(\cdot)$ can be related as it pertains to existence and stability/attractivity of positive equilibrium populations.

A key assumption in (1) is that density dependence only enters in via competition from possible recruits with other possible recruits (e.g. seeds vs. seeds). However, in many cases adult conspecifics can impose a negative density-dependent feedback on the establishment of recruits (Pico \& Retana, 2008; Silva Matos, Freckelton, \& Watkinson, 1999). For example, in perennial plant populations adult conspecifics can elicit a negative density-dependent feedback on seedling establishment through resource-limiting mechanisms such as shading. Therefore, in this paper we discuss the theoretical implications of weakening the assumption that density-dependent feedbacks are limited to feedbacks between possible recruits. To incorporate a negative feedback from adult conspecifics on recruitment we will assume that $f$ is a function of both the density of possible recruits, $y=c^{T} n_{t}$, and of some measurement of the amount of resources that are used by the adult population at time $t$, which we will call $z=d^{T} n_{t}$. Here, $d^{T}$ is a functional from $X$ to $\mathbb{R}^{+}$modelling the amount of resources that a possible recruit would use for establishment that is taken up by the adult population. The units of $d^{T}$ are those of the possible recruits (e.g. seeds) divided by those of the adult population (e.g. number or density of adult plants), so that $y+z$ can be added together. To incorporate the effect of adult conspecifics, we will assume that $f$ now takes the form

$$
f(y, z)=g(y+z) y
$$

where $g$ is the same establishment probability as before. It follows that $f(\cdot, z)$ is increasing, concave down in $y$ with $f(0, z)=0$ for each fixed $z$ and that $f(y, \cdot)$ is a decreasing function in $z$ for each fixed $y$. Examples of functions that incorporate these assumptions are a 
modified power function of the form

$$
f(y, z)=\frac{\beta y}{(z+y)^{1-a}} \quad \text { with } a \in(0,1) \text { and } \beta>0,
$$

or a modified Michaelis-Menten-type function of the form

$$
f(y, z)=\frac{\alpha y}{\beta+z+y} \quad \text { with } \alpha>0 \text { and } \beta>0 .
$$

With these new assumptions, (2) becomes

$$
n_{t+1}=A n_{t}+b f\left(c^{T} n_{t}, d^{T} n_{t}\right) .
$$

In the coming sections we study the asymptotic properties of the unique positive equilibrium population $n^{*}$ of this Luré system, and show the effects of including density dependence from adult conspecifics. We find that, for some $\left(c^{T}, d^{T}\right)$ combinations $n^{*}$ is globally attracting and/or globally asymptotically stable, but for other combinations the global properties of this equilibrium are still an open question. We apply these results to a published model from Rose et al. (2005).

\section{Abstract formulation}

We call $X$ the Banach space in which our population $n_{t}$ evolves. That is, the population $n_{t} \in X$ for all $t$. In the population project matrix case, $X=\mathbb{R}^{m}$, where $m$ is the number of discrete life-history stages. In the integral project model case $X=L^{1}(L, U)$, where the interval $(L, U)$ is the range of the population's continuous life-history stage variable. We only consider the cases where $X=\mathbb{R}^{m}$ or $X=L^{1}(L, U)$. Since $n_{t}$ is a population for each $t$, we wish to work with non-negative vectors in $X$ and non-negative operators on $X$. Let $K \subset X$ be a reproducing cone inducing a partial order $\geq$ on $X$, where $x \geq y$ means that $x-y \in K$. If $x \geq 0$, i.e. $x$ is in $K$, we say that $x$ is a non-negative vector. An example of a nonnegative vector in $\mathbb{R}^{m}$ is a vector with all nonnegative elements and an example of a nonnegative vector in $L^{1}(L, U)$ is a function that is nonnegative for almost every element in $(L, U)$.

An operator on $X$ is considered a non-negative operator if it maps non-negative vectors to non-negative vectors. For example, when $X=\mathbb{R}^{m}$, an $m \times m$ matrix $M$ is a non-negative operator on $X$ if and only if all of its entries are non-negative.

For our model (5) we need some assumptions on the triple $\left(A, b, c^{T}\right)$, as well as $d^{T}$ and $f$. These assumptions are natural when modelling structured population dynamics. These assumptions are given by the following:

(A1) $A \in \mathcal{L}(X)$ is a non-negative operator with spectral radius $r(A)<1$;

(A2) $b$ is a non-negative vector in $X$;

(A3) If $X=L^{1}(L, U), c^{T}: X \rightarrow \mathbb{R}$ is a non-negative functional in the sense that

$$
c^{T} n \geq 0, \text { for all } n \geq 0
$$

or, if $X=\mathbb{R}^{m}$, the matrix $A+p b c^{T}$ is a primitive matrix for all $p>0$; 
(A4) $d^{T}: X \rightarrow \mathbb{R}$ is a non-negative functional in the sense that

$$
d^{T} n \geq 0, \text { for all } n \geq 0
$$

Additionally,

$$
d^{T}(I-A)^{-1} b>0 .
$$

(A5) The function $g: \mathbb{R}^{+} \rightarrow \mathbb{R}^{+}$is a $C^{1}$ and strictly decreasing function such that $\lim _{y \rightarrow \infty} g(y)=0$. The the function $f(y, z)=g(y+z) y$ is increasing and concave down in $y$ for each $z$.

Biologically, (A1) prevents the existence of immortal individuals (i.e. individuals with infinite life expectancy). (A2) states that the distribution of newborns' lives in the same space as the population itself. (A3) states that the production of possible recruits is nonnegative in the $X=L^{1}(L, U)$ case, while in the $\mathbb{R}^{m}$ case such that there exists a $t>0$ where the matrix $\left(A+p b c^{T}\right)^{t}$ is a strictly positive for all $p>0$. The former assumption is the minimum assumption necessary for a population model, while the latter is one that is imposed on many of the linear matrix models in the literature (Stott, Townley, Carslake, \& Hodgson, 2010). The positivity of $\left(A+p b c^{T}\right)^{t}$ for some $t$ ensures that individuals in every stage can eventually contribute individuals to every other stage. (A4) States that the equilibrium stage distribution $(I-A)^{-1} b$ elicits some negative density-dependent feedback on recruitment, and that this density-dependent recruitment is nonnegative. Finally, (A5) guarantees that the establishment probability decreases as the density of possible recruits and/or adult conspecifics increases, and as the possible recruits/adult conspecifics becomes arbitrarily large, the probability of any one of the possible recruits establishing will become arbitrarily small. The assumptions on $g$ imply also that $f(0, z)=0$ for each $z$ and $f$ is a decreasing function of $z$ for each $y$.

What distinguishes the setting in this paper from that in Rebarber et al. (2012) and Townley et al. (2012) is that the nonlinear feedback

$$
g(y+z) y=f(y, z)
$$

is not a monotone increasing function in population size: certain increases in the population $n_{t}$ will elicit population decline while others will elicit population increase. This is in great contrast to the results in Rebarber et al. (2012), Townley et al. (2012), and subsequent work (Eager et al., 2014a; Smith \& Thieme, 2013). In the next section we show how this non-monotonicity alters the stability results we see in those references.

\section{Equilibrium results}

In this section, we present results on the persistence, global asymptotic stability, global attractivity and asymptotic stability of the population $n_{t}$ modelled by (5). Theorem 3.1 shows that if the stability radius $p_{e}$ exceeds the largest possible establishment probability $g_{0}$, the extinction state $n_{t}=0$ is globally asymptotically stable, and the population dies out regardless of initial population. Biologically, this means that if the establishment probability in the best-possible, density-independent scenario is not enough to push the spectral radius of the operator $A+p b c^{T}$ above unity, than the population will decay at a rate less than the spectral radius of $A+p_{e} b c^{T}$ 
Theorem 3.2 shows that, if $g_{0}>p_{e}$ and the functional $d^{T}$ modelling competition from adult conspecifics is a constant multiple of the functional $c^{T}$ modelling the production of possible recruits, then (5) has a globally asymptotically stable equilibrium population $n^{*}$. When $p_{e}<g_{0}$ the equilibrium population $n^{*}$ and the stability radius $p_{e}$ solve the system of equations

$$
\begin{aligned}
& n^{*}=A n^{*}+p_{e} b c^{T} n^{*} \\
& p_{e}=g\left(c^{T} n^{*}+d^{T} n^{*}\right),
\end{aligned}
$$

yielding

$$
n^{*}=\frac{p_{e} g^{-1}\left(p_{e}\right)}{1+p_{e} p_{d}^{-1}}(I-A)^{-1} b
$$

where $p_{d}=\left(d^{T}(I-A)^{-1} b\right)^{-1}$ is the stability radius of the triple $\left(A, b, d^{T}\right)$ and $g^{-1}$ is the inverse of the function $g$, which exists due to its monotonicity.

Notice the the equilibrium population in (7) can be understood in a straightforward way ecologically: the vector $(I-A)^{-1} b=b+A b+A^{2} b+\cdots$ is the full evolution of the stage structure of a population that is initially distributed via the newborn distribution vector $b$. The constant $p_{e}$, as previously stated, is the long-term establishment probability for possible recruits, while

$$
\frac{g^{-1}\left(p_{e}\right)}{1+p_{e} p_{d}^{-1}}
$$

is the long-term production of possible recruits. The only difference between $n^{*}$ in (7) and that from Theorem 1.1 is the production term. The two become equal when $d^{T}=0$, and $n^{*} \rightarrow 0$ as $d^{T}$ increases (and $p_{d}$ subsequently decreases), as expected.

Theorem 3.3 states that if $g_{0}>p_{e}$ and

$$
r\left(A+p_{e} b c^{T}+b\left(d^{T}+c^{T}\right) g^{\prime}\left(g^{-1}\left(p_{e}\right)\right) \frac{g^{-1}\left(p_{e}\right)}{1+p_{e} p_{d}^{-1}}\right)<1,
$$

then $n^{*}$ given by (7) is an asymptotically stable population. The linear operator in (8) can be thought of as the operator

$$
A+p_{e} b c^{T}
$$

which has spectral radius equal to unity, minus the operators

$$
-b d^{T} g^{\prime}\left(g^{-1}\left(p_{e}\right)\right) \frac{g^{-1}\left(p_{e}\right)}{1+p_{e} p_{d}^{-1}}
$$

and

$$
-b c^{T} g^{\prime}\left(g^{-1}\left(p_{e}\right)\right) \frac{g^{-1}\left(p_{e}\right)}{1+p_{e} p_{d}^{-1}},
$$

which is the sum of the density-dependent influences on recruitment from a) density of adult conspecifics and $b$ ) the density possible recruits. 
Additionally, if $p_{d}>p_{e}$ then the population $n^{*}$ given by (7) is also a globally attracting equilibrium population, which coupled with (8), makes it a globally asymptotically stable equilibrium population. Biologically, $p_{d}>p_{e}$ means that

$$
d^{T}(I-A)^{-1} b<c^{T}(I-A)^{-1} b,
$$

which implies that competition from the equilibrium distribution $v=(I-A)^{-1} b$ of adult conspecifics is weaker than the equilibrium production of (and competition elicited by) possible recruits. If this is the case, then $n^{*}$ from (7) is globally attracting, and globally asymptotically stable if (8) additionally holds. We present these results formally below.

Theorem 3.1: Suppose (A1), (A2), (A3), (A4) and (A5) hold and $p_{e}>g_{0}$, then the zero vector is a globally asymptotically stable equilibrium for (5) in the sense that, for every $n_{0} \in K$,

$$
\lim _{t \rightarrow \infty} n_{t}=0
$$

Futhermore, for every $\epsilon>0$, there exists a $\delta>0$ such that $\left\|n_{0}\right\|<\delta$ implies that $\left\|n_{t}\right\|<\epsilon$ for all $t \in \mathbb{N}$.

Proof: If $p_{e}<g_{0}$, then

$$
n_{t+1}=A n_{t}+b g\left(c^{T} n_{t}+d^{T} n_{t}\right) c^{T} n_{t} \leq A n_{t}+p b c^{T} n_{t}
$$

for some $p \leq p_{e}$ which, by induction, means that

$$
n_{t} \leq\left(A+p b c^{T}\right)^{t} n_{0}
$$

Since $r\left(A+p b c^{T}\right)<1$ we have that $\lim _{t \rightarrow \infty} n_{t}=0$. The boundedness of $\left(A+p b c^{T}\right)$ implies the last statement of the result.

Theorem 3.2: Suppose (A1), (A2), (A3), (A4) and (A5) hold and $p_{e}<g_{0}$. Assume further that there exists a $\gamma$ such that $d^{T}=\gamma c^{T}$. Then the vector $n^{*}$ from (7) is a globally asymptotically stable equilibrium of (5) in $K \backslash\{0\}$ in the sense that

$$
\lim _{t \rightarrow \infty} n_{t}=n^{*}
$$

for any $n_{0} \in K \backslash\{0\}$, and for every $\epsilon>0$, there exists a $\delta>0$ such that $\left\|n_{0}-n^{*}\right\|<\delta$ implies that $\left\|n_{t}-n^{*}\right\|<\epsilon$ for all $t \in \mathbb{N}$.

Proof: The result follows directly from Theorem 3.3 in Rebarber et al. (2012) and Theorem 1.1 in Townley et al. (2012), since if $f(y)=g(y) y$ is increasing and concave down in $y$ with $f(0)=0$, then so is $f(y, \gamma y)=g((1+\gamma) y) y$.

Theorem 3.3: Suppose (A1), (A2), (A3), (A4) and (A5) hold and $p_{e}<g_{0}$. If the specral radius of the operator

$$
A+p_{e} b c^{T}+b\left(d^{T}+c^{T}\right) g^{\prime}\left(g^{-1}\left(p_{e}\right)\right) \frac{g^{-1}\left(p_{e}\right)}{1+p_{e} p_{d}^{-1}}
$$

is less than unity, the vector $n^{*}$ from (7) is a locally asymptotically stable equilibrium of (5) in $K \backslash\{0\}$. 
If $p_{e}<p_{d}$, then the vector $n^{*}$ from (7) is a globally attracting equilibrium of (5) in $K \backslash\{0\}$ in the sense that

$$
\lim _{t \rightarrow \infty} n_{t}=n^{*}
$$

for any $n_{0} \in K \backslash\{0\}$.

If $p_{e}<p_{d}$ and the spectral radius of the operator (9) is smaller than unity, then $n^{*}$ from (7) is a globally asymptotically stable equilibrium of (5) in $K \backslash\{0\}$ in the sense that

$$
\lim _{t \rightarrow \infty} n_{t}=n^{*}
$$

for any $n_{0} \in K \backslash\{0\}$, and for every $\epsilon>0$, there exists a $\delta>0$ such that $\left\|n_{0}-n^{*}\right\|<\delta$ implies that $\left\|n_{t}-n^{*}\right\|<\epsilon$ for all $t \in \mathbb{N}$.

Proof: To prove the first part we know that, for any $p_{e}, p_{d}$ such that $p_{e}<g_{0}$, linearizing (5) about the equilibrium population $n^{*}$ from (7) yields the following operator

$$
A+b g\left(c^{T} n^{*}+d^{T} n^{*}\right) c^{T}+b g^{\prime}\left(c^{T} n^{*}+d^{T} n^{*}\right)\left(c^{T} n^{*}\right)\left(c^{T}+d^{T}\right)
$$

which reduces to the operator (9). The local asymptotic stability of $n^{*}$ is achieved when the spectral radius of (9) is smaller than unity (Townley et al., 2012), completing the proof.

To prove the second part we start by successive approximation using the results from Rebarber et al. (2012) and Townley et al. (2012), which covers the $X=L^{1}(L, U)$ case and Townley et al. (2012), which covers the $X=\mathbb{R}^{m}$ case. In both cases, the fact that $g$ is decreasing means that, for any $t \in \mathbb{N}$,

$$
n_{t+1}=A n_{t}+b f\left(c^{T} n_{t}, d^{T} n_{t}\right) \leq A n_{t}+b f\left(c^{T} n_{t}, 0\right)
$$

The rightmost part of (10) is the right-hand side of the model (2), whose global asymptotic stability is the subject of Theorem 1.1. Thus, we define $\bar{n}_{t}$ to be the population with the same initial condition as that modelled with (5) solving

$$
\bar{n}_{t+1}=A \bar{n}_{t}+b f\left(c^{T} \bar{n}_{t}, 0\right) .
$$

Since $g_{0}>p_{e}$, we have that

$$
\bar{n}_{0}^{*}=p_{e} g^{-1}\left(p_{e}\right)(I-A)^{-1} b
$$

is the globally asymptotically stable equilibrium vector of (11). Additionally, we know that, for every $\epsilon>0$ we have, for large enough $t$,

$$
n_{t} \leq \bar{n}_{0}^{*}+\frac{\epsilon}{0+1} \mathbb{1}
$$

where $\mathbb{1}$ is the vector with a 1 in every component (in the $\mathbb{R}^{m}$ case), or the function that's equal to 1 everywhere (in the $L^{1}(L, U)$ case). Thus, by the monotonicity of $g$ we have

$$
n_{t+1}=A n_{t}+b f\left(c^{T} n_{t}, d^{T} n_{t}\right) \geq A n_{t}+b f\left(c^{T} n_{t}, d^{T}\left(\bar{n}_{0}^{*}+\frac{\epsilon}{0+1} \mathbb{1}\right)\right) .
$$


The right-hand side of (12) is simply another version of the model (2), with $g(y)=$ $\left.g\left(y+d^{T} \bar{n}_{0}^{*}+\frac{\epsilon}{0+1} \mathbb{1}\right)\right)$, since $d^{T}\left(\bar{n}_{0}^{*}+\frac{\epsilon}{0+1} \mathbb{1}\right)$ is simply a constant and $f\left(y, d^{T}\left(\bar{n}_{0}^{*}+\frac{\epsilon}{0+1} \mathbb{1}\right)\right)$ is increasing, concave down, with $f\left(0, d^{T}\left(\bar{n}_{0}^{*}+\frac{\epsilon}{0+1} \mathbb{1}\right)\right)=0$. Theorem 1.1 states that the population $\underline{n}_{t}$ that solves

$$
\underline{n}_{t+1}=A \underline{n}_{t}+b f\left(c^{T} \underline{n}_{t}, d^{T}\left(\bar{n}_{0}^{*}+\frac{\epsilon}{0+1} \mathbb{1}\right)\right)
$$

has a non-zero, globally asymptotically stable equilibrium vector if

$$
\underline{g}_{0}=g\left(0+d^{T}\left(\bar{n}_{0}^{*}+\frac{\epsilon}{0+1} \mathbb{1}\right)\right)>p_{e},
$$

which can be rewritten as

$$
g\left(p_{e} g^{-1}\left(p_{e}\right) d^{T}(I-A)^{-1} b+\frac{\epsilon}{1+0} d^{T} \mathbb{1}\right)=g\left(p_{e} g^{-1}\left(p_{e}\right) p_{d}^{-1}+\frac{\epsilon}{1+0} d^{T} \mathbb{1}\right)>p_{e}
$$

which reduces to

$$
p_{e}+\left(\frac{p_{d} d^{T} \mathbb{1}}{g^{-1}\left(p_{e}\right)}\right) \epsilon<p_{d}
$$

Since $\epsilon>0$ was arbitrary, in the case where $p_{e}<p_{d}$, (13) has a positive, globally asymptotically stable equilibrium vector $\underline{n}_{0}^{*}$. Additionally, we know that, for every $\epsilon>0$ we have, for large enough $t$

$$
n_{t} \geq \underline{n}_{0}^{*}-\frac{\epsilon}{0+1} \mathbb{1}
$$

From $\underline{n}_{0}^{*}$ we can create a new model

$$
\bar{n}_{t+1}=A \bar{n}_{t}+b f\left(c^{T} \bar{n}_{t}, d^{T}\left(\underline{n}_{0}^{*}-\frac{\epsilon}{0+1} \mathbb{1}\right)\right)
$$

with the same initial condition as that modelled with (5). This is simply another version of the model (2) which, by the monotonicity of $g$, is also larger than $n_{t}$ from (5) for all $t$. The globally stable equilibrium $\bar{n}_{1}^{*}$ of this new model is such that $\bar{n}_{1}^{*}<\bar{n}_{0}^{*}$, by the fact that, for sufficiently small $\epsilon, d^{T}\left(\underline{n}_{0}^{*}-\frac{\epsilon}{0+1} \mathbb{1}\right)>0$ (by A4) and $g$ is decreasing. We can then use $\bar{n}_{1}^{*}$ to create another new model

$$
\underline{n}_{t+1}=A \underline{n}_{t}+b f\left(c^{T} \underline{n}_{t}, d^{T}\left(\bar{n}_{1}^{*}+\frac{\epsilon}{1+1} \mathbb{1}\right)\right),
$$

which is smaller than $n_{t}$ from (5) for all $t$, and whose globally stable equilibrium $\underline{n}_{1}^{*}$ is such that $\underline{n}_{1}^{*}>\underline{n}_{0}^{*}$, by the fact that $\bar{n}_{1}^{*}<\bar{n}_{0}^{*}$ and $g$ is decreasing. If we continue in this way, we can build two sequences $\left\{\bar{n}_{j}^{*}\right\}_{j=0}^{\infty}$ and $\left\{\underline{n}_{j}^{*}\right\}_{j=0}^{\infty}$, where the former sequence is decreasing in and the latter is increasing. Additionally, for each $j$ we have that, for sufficiently large $t$ :

$$
\underline{n}_{j}^{*}-\frac{\epsilon}{j+1} \mathbb{1} \leq n_{t} \leq \bar{n}_{j}^{*}+\frac{\epsilon}{j+1} \mathbb{1} .
$$

Since $\left\{\bar{n}_{j}^{*}\right\}_{j=0}^{\infty}$ and $\left\{\underline{n}_{j}^{*}\right\}_{j=0}^{\infty}$ are monotone sequences, they both have a limit, which we will name $\bar{n}^{* *}$ and $\underline{n}^{* *}$, respectively. By the continuity of $g$, the former limit solves the following 
fixed-point equation

$$
\bar{n}^{* *}=A \bar{n}^{* *}+\operatorname{bg}\left(c^{T} \bar{n}^{* *}+d^{T} \underline{n}^{* *}\right) c^{T} \bar{n}^{* *},
$$

while the latter solves

$$
\underline{n}^{* *}=A \underline{n}^{* *}+b g\left(c^{T} \underline{n}^{* *}+d^{T} \bar{n}^{* *}\right) c^{T} \underline{n}^{* *} .
$$

Solving for $\bar{n}^{* *}$ and $\underline{n}^{* *}$ yield

$$
\bar{n}^{* *}=p_{e}\left(g^{-1}\left(p_{e}\right)-d^{T} \underline{n}^{* *}\right)(I-A)^{-1} b,
$$

and

$$
\underline{n}^{* *}=p_{e}\left(g^{-1}\left(p_{e}\right)-d^{T} \bar{n}^{* *}\right)(I-A)^{-1} b .
$$

As we discussed previously in the introduction, the only way the models (11) and (13) and their subsequent modifications equilibrate is if

$$
g\left(c^{T} \bar{n}^{* *}+d^{T} \underline{n}^{* *}\right)=p_{e}
$$

and

$$
g\left(c^{T} \underline{n}^{* *}+d^{T} \bar{n}^{* *}\right)=p_{e}
$$

respectively. Since $g$ is monotone, this can only happen if

$$
c^{T} \bar{n}^{* *}+d^{T} \underline{n}^{* *}=c^{T} \underline{n}^{* *}+d^{T} \bar{n}^{* *},
$$

or, using (16) and (17),

$$
g^{-1}\left(p_{e}\right)-d^{T} \underline{n}^{* *}+p_{e} p_{d}^{-1}\left(g^{-1}\left(p_{e}\right)-d^{T} \bar{n}^{* *}\right)=g^{-1}\left(p_{e}\right)-d^{T} \bar{n}^{* *}+p_{e} p_{d}^{-1}\left(g^{-1}\left(p_{e}\right)-d^{T} \bar{n}^{* *}\right)
$$

or

$$
d^{T} \underline{n}^{* *}=d^{T} \bar{n}^{* *}
$$

It follows that the models

$$
\bar{n}_{t+1}=A \bar{n}_{t}+b f\left(c^{T} \bar{n}_{t}, d^{T} \underline{n}^{* *}\right)
$$

and

$$
\underline{n}_{t+1}=A \underline{n}_{t}+b f\left(c^{T} \underline{n}_{t}, d^{T} \bar{n}^{* *}\right)
$$

both have $n^{*}$ from (7) as its unique, positive, globally stable equilibrium population. Therefore,

$$
\lim _{t \rightarrow \infty} n_{t}=n^{*}
$$

as sought.

To prove the third part we note that, if $p_{e}<p_{d}$ and the spectral radius of (9) is less than unity, the global asymptotic stability of $n^{*}$ follows from the previous two proofs. 


\section{Example}

We now apply our results to the Platte thistle (Cirsium canescens) model found in Rose et al. (2005) and reformulated in Eager et al. (2012). Let $n(x, t)$ be the function such that

$$
\int_{x}^{x+\delta x} n(x, t) \mathrm{d} x
$$

gives the total number of individuals in the thistle population with stage variable in the interval $[x, x+\delta x]$. In Rose et al. (2005), the stage variable $x$ models the natural log of the root crown diameter of the thistle, at time $t$, which is in years. We assume that $x$ is in the interval $(-2,5.5)$, and thus $X=L^{1}(-2,5.5)$ is our state space. The survival and growth kernel consists of the probability $s_{p}(x)$ that an individual of size $x$ survives a given time-step times the probability $g(x, y)$ that an individual of size $x$ grows to an individual of size $y$ in one time-step. Finally, since the Platte thistle is a monocarpic plant, we also need to include the probability that a given thistle does not flower, which we denote with $1-f_{p}(x)$. If survival, growth and reproduction are probabilistically independent, we can give the operator $A$ as

$$
A u=\int_{-2}^{5.5} k(\cdot, x) u(x) \mathrm{d} x=\int_{-2}^{5.5}\left(1-f_{p}(x)\right) s_{p}(x) g(\cdot, x) u(x) \mathrm{d} x
$$

for $u \in L^{1}(-2,5.5)$. The vector $b$ is given by the normal probability distribution $J(x)$ in Rose et al. (2005) modelling the distribution of juveniles in their first year. Finally, to create the functional $c^{T}$ we assume that an individual needs to survive to reproduce. We thus multiply this survival probability $s_{p}(x)$ by the probability of reproduction $f_{p}(x)$ times the density of seed created $S(x)$ to make the functional $c^{T}$, which has the representation

$$
c^{T} u=\int_{-2}^{5.5} c(x) u(x) \mathrm{d} x=\int_{-2}^{5.5} s_{p}(x) f_{p}(x) S(x) u(x) \mathrm{d} x
$$

for $u \in L^{1}(-2,5.5)$. If follows from Rebarber et al. (2012) that the triple $\left(A, b, c^{T}\right)$, using the functions given in Rose et al. (2005) satisfy the assumptions (A1), (A2) and (A3). For additional biological background on Cirsium canescens, including modelling assumptions, please see pages $454-455$ in Rose et al. (2005).

In Eager et al. (2012) we discussed the implications of using a power function

$$
f(y, z)=\frac{\beta y}{(z+y)^{1-a}}
$$

for the nonlinear fecundity function in (5) in lieu of a more realistic function like a Michaelis-Menton function. Due to the pathological biological implications of using a power function, which include allowing for unbounded establishment probabilities, we 
will use the Michaelis-Menton function

$$
f(y, z)=\frac{\alpha y}{\beta+y+z}
$$

for $f$ in this example. Here, when $\alpha, \beta>0$, the establishment probability $g(y)=\alpha(\beta+y)^{-1}$, as well as the total recruitment function $f$, satisfy the assumption (A5). We assume that $\alpha$ and $\beta$ are the same parameter values found in Eager et al. (2012).

To account for competition from adult conspecifics, we assume that the functional $d^{T}$ is modelled by the integral

$$
d^{T} u=\int_{-2}^{5.5} d(x) u(x) \mathrm{d} x=\int_{-2}^{5.5} s_{p}(x)\left(1-f_{p}(x)\right) D(x) u(x) \mathrm{d} x .
$$

Thus, we are assuming that only the adult plants that survive and don't reproduce compete with possible recruits in their path towards establishment. We assume that $D$ is an increasing function of $x$. That is, the larger the root crown diameter of the thistle, the stronger the competitive feedback. Assume for simplicity that $D$ is a linear function of $x$ :

$$
D(x)=\delta(x+2)
$$

For $\delta>0, d^{T}$ satisfies the assumption (A4), and we can apply the results in Theorems 3.1 and 3.3. Using a convex function for $D$ would cause $p_{d}$ to decrease and hence lower our chances of being able to use Theorem 3.3 to analyse the stability/attractivity of $n^{*}$. The opposite would be true if we used a concave function for $D$, as $p_{d}$ would increase in that case. Since $d^{T}$ is not a constant multiple of $c^{T}$, it follows that we cannot use the result from Theorem 3.2.

For the parameter values from Rose et al. (2005) and Eager et al. (2012), we have that

$$
p_{e}=.0216 \text { and } \quad g_{0}=\frac{\alpha}{\beta}=\frac{219.6}{756.7}=.2902
$$

Since $g_{0}>p_{e}$, the population $n(x, t)$ is persistent in the sense of Smith and Thieme (2013) (also see Theorem 6.8 in Wen, Smith, \& Thieme, 2015), and thus the population does not go extinct as $t \rightarrow \infty$. Additionally, the stability radius of the linear data $\left(A, b, d^{T}\right)$ is, as a function of $\delta$, equal to

$$
p_{d}=.7155 \delta^{-1}
$$

Figure 1 displays the spectral radius of

$$
A+p_{e} b c^{T}+b\left(d^{T}+c^{T}\right) g^{\prime}\left(g^{-1}\left(p_{e}\right)\right) \frac{g^{-1}\left(p_{e}\right)}{1+p_{e} p_{d}^{-1}}
$$

as a function of $\delta$. Notice that the spectral radius appears to be bounded above by unity, implying at least the asymptotic stability of the equilibrium population $n^{*}(x)$, whose distribution is given by Figure 2. Theorem 3.3 implies the global attractivity (and thus the global asymptotic stability) of $n^{*}(x)$ if $\delta<33.1173$. Thus, it would appear that the 


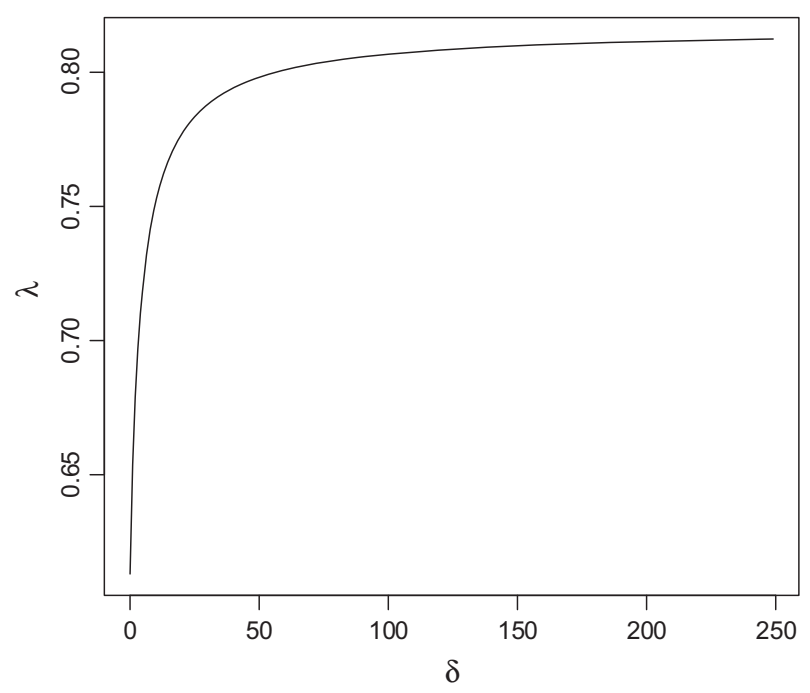

Figure 1. The spectral radius of the linear operator (9) found from linearizing the model (5) applied to the Platte thistle (Cirsium canescens), using data from Rose et al. (2005) and Eager et al. (2012), as a function of the strength $\delta$ of density-dependent feedback from adult conspecifics.

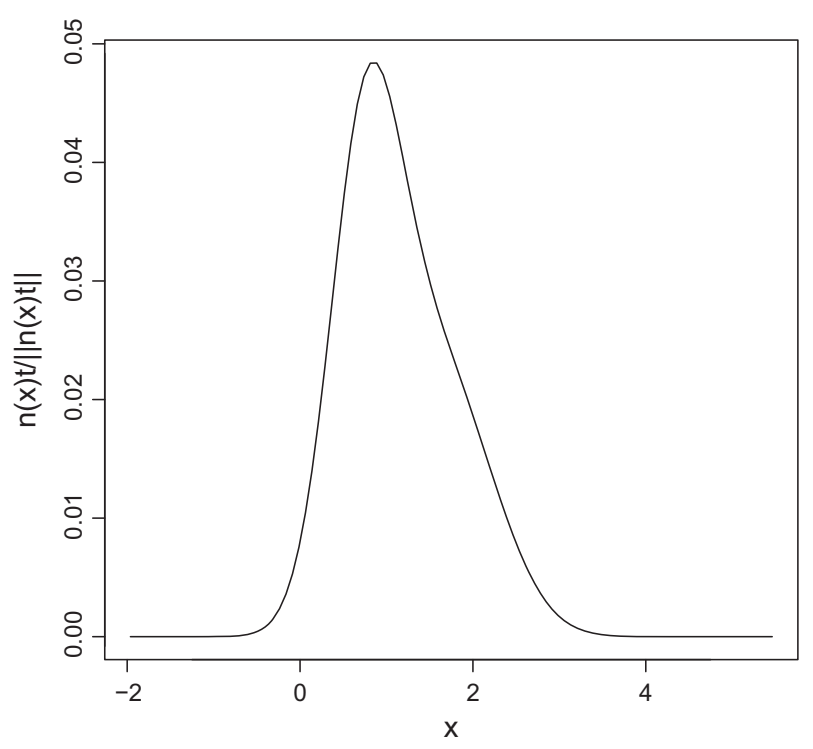

Figure 2. The size distribution of the equilibrium population $n^{*}$ from (5) applied to the Platte thistle (Cirsium canescens), using data from Rose et al. (2005) and Eager et al. (2012). This distribution is proportional to the function $(I-A)^{-1} b$ and is scaled to integrate to unity.

average thistle would need to have a substantial competitive feedback on establishing seeds for the global asymptotic stability of the equilibrium population $n^{*}$ to be in question.

Figure 3 displays trajectories of the total population size $\|n(x, t)\|_{1}$ under the following scenarios for $\delta: 1,25,50,100$. Notice that, even in the case where $\delta \geq 33.1173$, it appears 
(a)

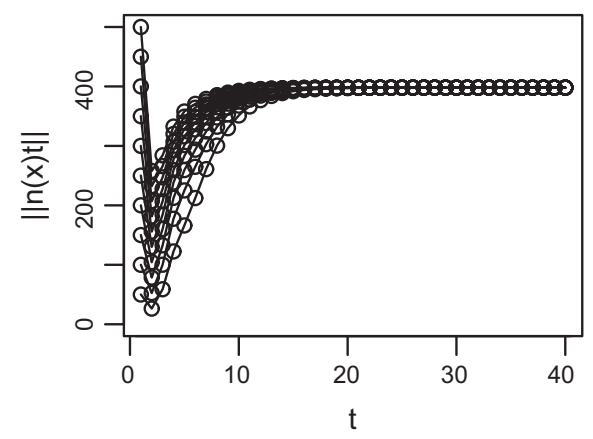

(c)

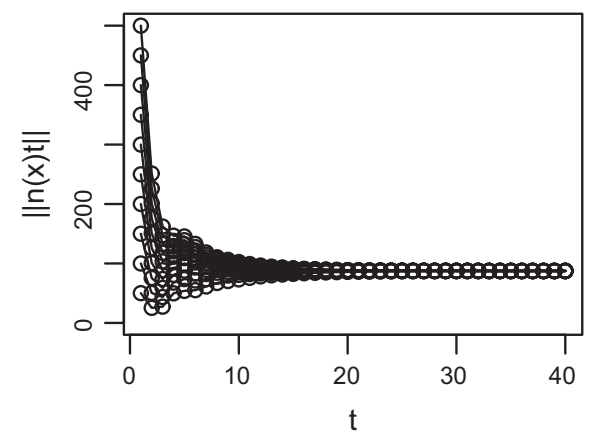

(b)

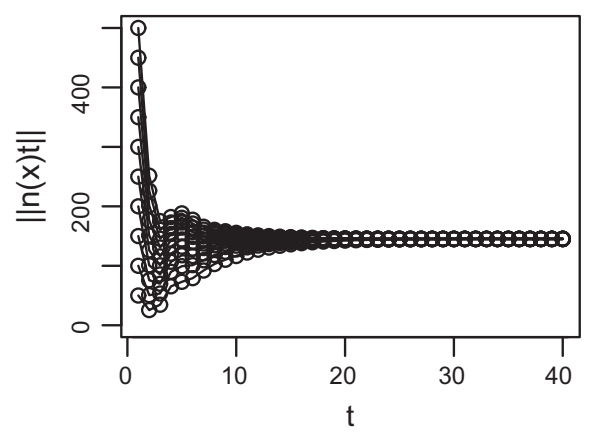

(d)

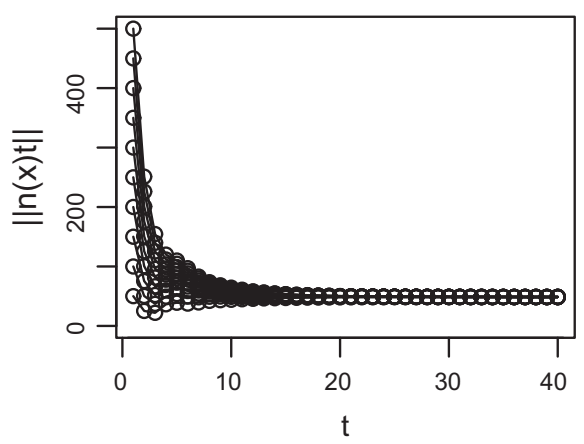

Figure 3. Above are trajectories of the model (5) applied to the Platte thistle (Cirsium canescens), using data from Rose et al. (2005) and Eager et al. (2012). We assumed that the initial population $n_{0}=N_{0} b$, where $b$ is the newborn distribution function and $N_{0}$ ranges from 50 to 500 in increments of 50 . Values of the strength of density-dependent feedback from adult conspecifics were (a) $\delta=1$, (b) $\delta=25$, (c) $\delta=50$ and (d) $\delta=100$.

that the population size still converges to $\left\|n^{*}(x)\right\|_{1}$, independent of initial population $n_{0}(x)$, with substantial transient dynamics in each case.

\section{Discussion}

In this paper, we studied the equilibrium dynamics of a Lur'e system modelling a structured population, where adult conspecifics were assumed to have a negative density-dependent feedback on the recruitment of newborns. The assumption of feedback from adult conspecifics elicits models that do not have the monotonicity exploited in Rebarber et al. (2012), Townley et al. (2012), Smith and Thieme (2013), Eager et al. (2014a), with the stability results (at least for now) subsequently weakened. In cases where the population is persistent $\left(g_{0}>p_{e}\right)$, we are only able to obtain global attractivity of the non-zero equilibrium population when the strength of density-dependent feedbacks from adult conspecifics are less than that from possible recruits themselves. When the relationship is flipped, i.e. $p_{e}>p_{d}$, the first lower approximation in the proof of Theorem 3.3 will always be 0 , leaving the upper approximation equal to

$$
\bar{n}^{*}=p_{e} g^{-1}\left(p_{e}\right)(I-A)^{-1} b
$$


for all successive iterations of the approximation scheme, which will not yield eventual convergence to the equilibrium population $n^{*}$. In Wen et al. (2015) they showed that population models like (5) are uniformly strongly persistent for all $p_{e}<g_{0}$ (including $\left.p_{e}>p_{d}\right)$. However, only the existence of a uniform lower bound is given, which is not enough to ensure that the successive approximations in the proof of Theorem 3.3 converge to each other, which is necessary to obtain global attractivity using this technique. Thus, while a clear extinction/persistence bifurcation occurs when $g_{0}$ passes through $p_{e}$ (or $n_{R} g_{0}$ passes through 1), the presence of persistence does not always help in the proof of attractivity or global asymptotic stability of positive equilibria.

To obtain asymptotic stability we needed to assume that the spectral radius of the operator (9) was less than unity. In exploratory simulations, we have yet to find a scenario where $g_{0}>p_{e}$ and this spectral radius is larger than unity. The fact that $g$ is decreasing means that the operator (9) is not always positive, which keeps us from using the powerful techniques generally exploited by theoretical ecologists (Caswell, 2001; Cushing, 1998; Lubben, 2009). Additionally, the presence of both $c^{T}$ and $d^{T}$ in (9) do not allow for a simple implementation of results like Theorem 2.4 in Smith and Thieme (2013). Such results are generally necessary but not sufficient, meaning that there are $\left(A, b, c^{T}\right), d^{T}$ and $f$ such that the spectral radius of (9) is less than unity, but the methods in theorems like Theorem 2.4 in Smith and Thieme (2013) are unable to prove it. Despite our inability to prove the global asymptotic stability of $n^{*}$ in these cases, we conjecture that it holds for all $\left(A, b, c^{T}\right), d^{T}$ and $f$ such that $p_{e}<p_{d}$, a conjecture we will continue to investigate.

While the extinction/persistence threshold does not change, the inclusion of competitive feedbacks from adult conspecifics always decreased equilibrium populations, which can be seen by comparing the formulas for $n^{*}$ found in Theorems 1.1 and 3.3. A closed-form formula for the equilibrium population, as in (7) has the benefit of being easily analysed using sensitivity and elasticity techniques. In Eager and Rebarber (2016) we rigorously derive sensitivity and elasticity formulas for Lur'e systems like (2). The model in this paper is only different in the reproduction term, and thus the only additions to the sensivity and elasticity formulas in Eager and Rebarber (2016) involve the derivatives of algebraic functions of $p_{e}$ and $p_{d}$, which amount to a straightfoward calculus exercise, as well as the derivative of $p_{d}$ with respect to $\left(A, b, c^{T}\right)$ and $d^{T}$, which are analogous to those for $p_{e}$.

Extensions of this work include the inclusion of competition from adult conspecifics in models accounting for multiple nonlinearities. For example, the model in Eager et al. (2014a) is a plant-seed bank model that has one nonlinear term for the establishment of of possible recruits and another for the production of possible recruits. While we were able to prove the global asymptotic stability of equilibirum populations for the models in that paper, investigations into the stability of equilibria when one includes feedbacks from adult conspecifics has thus far proven unfruitful due to the existence of multiple nonlinear equations more difficult than those in (6).

Another extension of this work includes the inclusion of stochasticity into models accounting for feedbacks from adult conspecifics. In Eager et al. (2014b), we show that a stochastic Lur'e system has a globally stable equilibrium measure either completely concentrated on the zero population or completely excluding the zero population. However, the results in that paper are tailored specifically to an integral projection model for disturbance specialist plants, and do not consider the extistence of density-dependent feedbacks from adult conspecifics. Other papers, for example Benaïm and Schreiber (2009), Schreiber, 
Benaïm, and Atchadé (2011), Schreiber (2012), provide exciting results regarding the population persistence, permanence and stability of equilibrium measures for stochastic models under relatively broad assumptions for the nonlinear terms modelling density dependence, albiet only for finite-dimensional models. Subsequent work will attempt to extend these persistence, permanence and stability results for stochastic analogues of (5), specifically accounting for feedbacks from adult conspecifics.

\section{Acknowledgements}

The author would like to thank Sebastian Schreiber for suggesting this problem in the fall of 2012 . The author would also like to thank Richard Rebarber for his extensive help with the ideas that eventually made it into this manuscript. Finally, the author would like to thank the two annonymous reviewers for their constructive suggestions that greatly improved this manuscript.

\section{References}

Bacaer, N. (2011). A short history of mathematical population dynamics. London: Springer-Verlag. Benaïm, M., \& Schreiber, S. J. (2009). Persistence of structured populations in random environments. Theoretical Population Biology, 76, 19-34.

Caswell, H. (2001). Matrix population models: Construction, analysis and interpretation (2nd ed.). New York, NY: Springer.

Caswell, H., Takada, T., \& Hunter, C. M. (2004). Sensitivity analysis of equilibrium in densitydependent matrix population models. Ecology Letters, 7, 380-387.

Cushing, J. M. (1998). An introduction to structured population dynamics. Philadelphia, PA: SIAM.

Eager, E. A., \& Rebarber, R. (2016). Sensitivity and elasticity analysis of a Lure system used to model a population subject to density-dependent reproduction. submitted.

Eager, E. A., Rebarber, R., \& Tenhumberg, B. (2012). Choice of density-dependent seedling recruitment function affects predicted transient dynamics: A case study with Platte thistle. Theoretical Ecology, 5, 387-401.

Eager, E. A., Rebarber, R., \& Tenhumberg, B. (2014a). Global asymptotic stability of plant seed bank models. Journal of Mathematical Biology, 69, 1-37.

Eager, E. A., Rebarber, R., \& Tenhumberg, B. (2014b). Modeling and analysis of a density-dependent stochastic integral projection model for a disturbance specialist plant and its seed bank. Bulletin of Mathematical Biology, 76, 1809-1834.

Easterling, M. R., Ellner, S. P., \& Dixon, P. M. (2000). Size-specific sensitivity: Applying a new structured population model. Ecology, 81, 694-708.

Hinrichsen, D., \& Pritchard, A. J. (2005). Mathematical systems theory I: Modeling, state space analysis, stability and robustness. New York, NY: Springer.

Lubben, J. (2009). Modeling and analysis of biological populations ( $\mathrm{PhD}$ thesis). University of Nebraska, Lincoln.

Pico, F. X., \& Retana, J. (2008). Age-specific, density-dependent and environment-based mortality of a short-lived perennial herb. Plant Biology, 10, 374-381.

Rebarber, R., Tenhumberg, B., \& Townley, S. (2012). Global asymptotic stability of density dependent integral population projection models. Theoretical Population Biology, 81, 81-87.

Rose, K. E., Louda, S. M., \& Rees, M. (2005). Demographic and evolutionary impacts of native and invasive insect herbivores on Cirsium canescens. Ecology, 46, 1048-1053.

Schreiber, S. J. (2012). Persistence for stochastic difference equations: A mini-review. Journal of Difference Equations and Applications (Special Issue on Stochastic Difference Equations), 18, 13811403.

Schreiber, S. J., Benaïm, M., \& Atchadé, K. A. S. (2011). Persistence in fluctuating environments. Journal of Mathematical Biology, 62, 655-683. 
Silva Matos, D. M., Freckelton, R. P., \& Watkinson, A. R. (1999). The role of density dependence in the population dynamics of a tropical palm. Ecology, 80, 2635-2650.

Smith, H. L., \& Thieme, H. R. (2013). Persistence and global stability for a class of discrete time structured population models. Discrete and Continuous Dynamical Systems, 33, 4627-4646.

Stott, I., Townley, S., Carslake, D., \& Hodgson, D. J. (2010). On reducibility and ergodicity of population projection matrix models. Methods in Ecology and Evolution, 1, 242-252.

Townley, S., Tenhumberg, B., \& Rebarber, R. (2012). Feedback control systems analysis of density dependent population dynamics. Systems and Control Letters, 61, 309-315.

Wen, J., Smith, H. L., \& Thieme, H. R. (2015). Persistence versus extinction for a class of discrete-time structured population models. Journal of Mathematical Biology. doi:10.1007/s00285-015-0898-8 